\title{
10. Visualizing data: A lived experience
}

\author{
Jill Simpson
}

\begin{abstract}
Researching data visualization as a lived experience provides a perspective from which to explore its social life. Borrowing elements from feminist autobiographical research and critical making, this chapter uses the personal story of the design and circulation of a hand-drawn, small-data visualization depicting the author's experience of Obsessive Compulsive Disorder. By critically reflecting on the visualization's design and circulation, this chapter engages with wider academic debates about data visualizations' subjectivities. Furthermore, by interrogating notions of authenticity and honesty associated with hand drawing, it introduces the idea of a politics of hand-drawn visual representations of data.
\end{abstract}

Keywords: Critical data studies; Hand-drawn; Situated knowledge; Feelings; Lived experience

\section{Introduction}

Adopting a critical data studies perspective, this chapter explores the processes at work in the design and circulation of one particular data visualization. It draws on a case study documenting the experience of manually gathering and visualizing my own data, through the analogue medium of hand drawing. By borrowing from elements of critical making, a participatory method which combines the process of making with critical thinking (Ratto, 2011), it was possible to engage with similar decision-making processes to those that designers perform in the production of data-driven visualizations.

Exploring data visualization through this very personal and lived experience of its design and circulation has provided an opportunity to critically reflect on three important issues. Firstly, how slow, manual data

Engebretsen, M. and H. Kennedy (eds.), Data Visualization in Society. Amsterdam: Amsterdam University Press, 2020 DOI 10.5117/9789463722902_CH10 
gathering reveals the subjectivities inherent in all datasets (Crawford, 2013) and their visual representations. Secondly, the ways that images circulate online through social media and how images can be operationalized and repurposed. And thirdly, the politics of hand drawing visual representations of data in a field dominated by digital design.

These seemingly separate issues are connected through discussions of how feelings and emotions are integral to the design of data visualizations. The significance of feelings in people's engagement with complex charts and graphs has already been recognized within the field of critical data studies (see, for example, D'Ignazio \& Klein, 2016; Kennedy \& Hill, 2018). However, this chapter will also reflect on the ways that data visualizations can be felt by those who produce them and the people about whom they tell a story.

Using personal experience as a starting point from which to explore broader social and theoretical issues is part of the feminist tradition of autobiographical research (see, for example, Miller, 1991; Stanley, 1992). Stanley and Wise (1983) argue that drawing on personal experiences (of the researcher or the researched) makes it possible to put theory into context and in doing so, researchers can explain not only what is known, but how it has come to be known (Stanley \& Wise, 1983). Within a broad tradition of autobiographical feminist research I draw more specifically on Miller's definition of personal criticism (1991, p. 1). This, Miller describes, 'entails an explicitly autobiographical performance within the act of criticism' (p. 1). Here, Miller is talking in the context of cultural criticism. However, drawing on Stanley and Wise (1983), I believe that the same approach can be used to provide a contextual critique of data and its visualization.

I have suffered with an anxiety disorder called Obsessive Compulsive Disorder (OCD) for most of my life. The data visualization which is the focus of this case study represents my compulsions to check and recheck the same things over and over again. The motivation to quantify and visualize my experience of OCD was to help people, who might never have suffered from a mental illness, to understand the way it impacts people's everyday lives. However, as I quantified, analysed, and visualized the data I also began to see it as a form of critical making (Ratto, 2011), in which I was re-enacting the same conscious and unconscious decisions made by data visualization designers (D'Ignazio \& Klein, 2016), albeit on a much smaller scale.

Critical making combines critical thinking with the act of making something and involves participatory design and prototyping (Ratto, 2011). The method I used was not participatory in a group sense, nor was it limited to prototyping, having set out with the intention of producing a finished data visualization which could be published. In these ways it differs from 
Ratto's definition of critical making (2011, p. 253). However, the design work I undertook was informed by critical literature on data and its visualization, and it was an iterative and reflective process, both important elements of critical making (Ratto, 2011). Furthermore, in line with one particular aim of this methodological approach, through making I was able to connect process and theory in order to produce academic critique (Ratto, Wylie, \& Jalbert, 2014). Combining personal criticism and elements of critical making has provided an original approach from which to explore the lived experience of data visualizations.

\section{Visualizing mental illness}

Mental ill health tends not to produce visible physical symptoms on the body, making it largely invisible to others. This can make it hard for people to imagine the different ways a mental illness might negatively impact a person's life in the same way they might empathize with a physical disability. This was reflected in the words of a senior aide to the UK's prime minister, when the aide suggested that state-funded benefits should go to people who were really disabled, and not to those at home on medication for anxiety (BBC News, 2017). The aide was rightly criticized, yet while reading about what the aide had said I began to wonder whether data visualization might offer a medium through which to make the affects and effects of mental illness more visible to others.

In order to visualize my experience of OCD it was necessary first to quantify it. To do this I drew on Dear Data, a small-data, analogue art project by Giorgia Lupi and Stefanie Posavec (Dear Data, n.d.). By small-data, I mean data that are collected about a very small number of participants and include only a few variables. In the case of Dear Data each dataset comprised of only one person's data collected about a single topic. This is in contrast to big datasets which, for example, might be made up of tweets published by millions of people (boyd \& Crawford, 2012). Dear Data was a year-long project in which the artists manually gathered self-tracked data about themselves, and visualized them. Each week they decided on a topic together on which to collect their data. Examples include: a week of laughter, a week of doors, and a week of complaints (Dear Data, n.d.). At the end of every week they would hand draw a visualization of their data onto the front of a post card before posting it to one another (Dear Data, n.d.). Through the process of gathering and visualizing their personal data about everyday topics, they were able to learn about one 
another's lives. I was drawn to this project because of its warmth and contextual detail, qualities which are not commonly associated with quantitative data.

Influenced by Dear Data, I began to manually self-track my illness over the course of one day. The most obvious way to quantify my experience was to record my compulsions to check and recheck the same things over and over again. Every time I checked a door handle, the floor area around where I had been sitting or standing, or even the URL of my email login page I would write it down. Using pen and paper I recorded what it was that I was checking, how many times I checked it and if the compulsion was repeated a specific number of times. If the checking incident was particularly frustrating or distressing I would also record some contextual detail about how I was feeling at that moment, or what had prompted the incident, to help me remember it in more detail when I came to analyse the data.

With the data recorded I was able to read through it and begin to identify patterns and trends in my checking behaviour. Whilst reviewing the data I also began to think about what I wanted to communicate to people through the visualization. In order to increase awareness and understanding of OCD it was important to draw people's attention to the time and effort given over to my compulsions and how this impacts my day-to-day life. To achieve this, the visualization needed to convey the number and repetitive nature of the compulsions. The story I wanted to tell not only shaped the way I organized the data, but also the design of the visualization. Metaphors are an important design tool through which to make the message or concept which is being communicated visually clearer to the recipient (Ursyn, 2008). Utilizing this design strategy I designed the chart so that it resembled the shape of the human brain (see Figure 10.1). With this metaphor I aimed to communicate the idea of physicality, so as to represent mental ill health as an embodied and lived experience. The notion of physicality was also intended to encourage people to think about how mental illnesses are often invisible to others, yet can still be debilitating.

The lines which form the shape of the visualization do not carry meaning about the data. Instead they are an aesthetic design choice intended to mimic the lines visible on the surface of a brain, thus strengthening the visual metaphor. At the end of each line sits a bubble, with each bubble representing a separate incident of checking. Their positioning is purely aesthetic and as such it is not possible to read the visualization as a timeline. The colours of the bubbles relate to categories of checking, for example, checking door and drawer handles, or checking the floor area around where I had been 


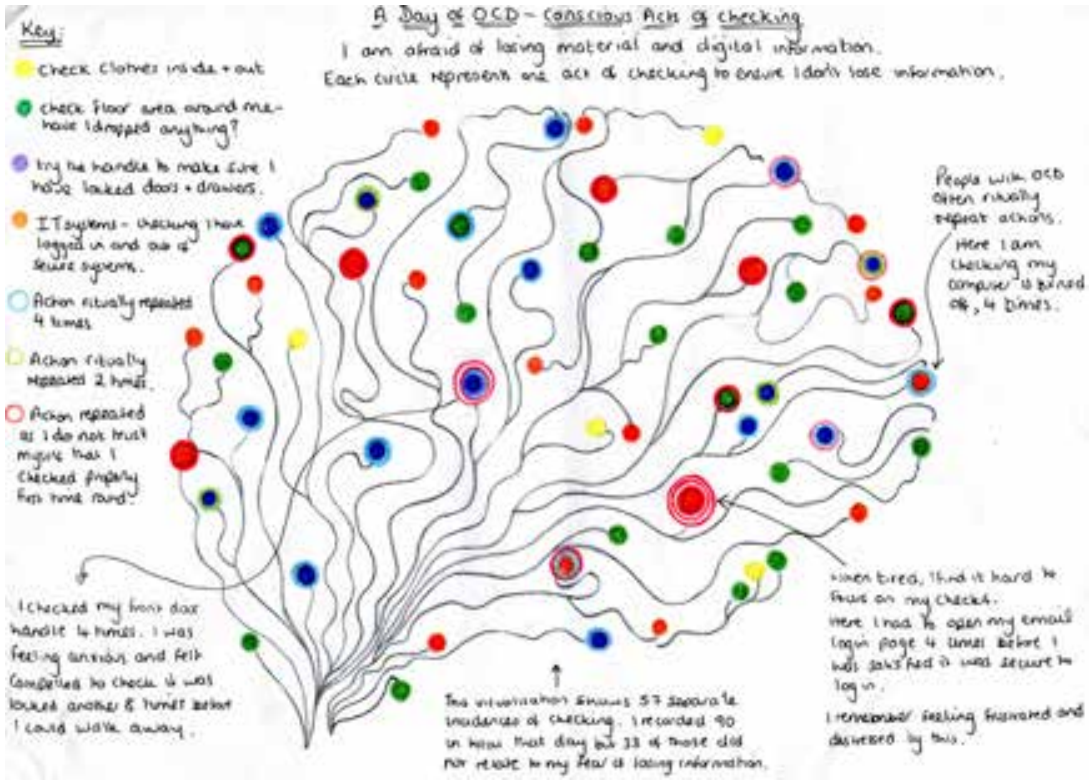

Figure 10.1. Visualizing mental illness: A day of OCD. Copyright 2017 by J. Simpson. Reprinted with permission.

standing or sitting down. Some of the bubbles have either a blue or green halo which indicates that the checking was ritually repeated a specific number of times, while a red halo represents when I felt compelled to repeat the action/s again. I chose bright colours and a recognizable shape as I wanted the visualization to be inviting and accessible. My aim to engage people who might never have experienced a mental illness led me to choose a pretty and whimsical design to encourage curiosity and to counter what I perceive to be negative cultural stereotypes about mental ill health.

Big data are critiqued for their lack of contextual detail (boyd \& Crawford, 2012; Kitchin, 2014). To help people connect with the individual behind the data, the visualization I designed included handwritten notes highlighting particular points in the data and explaining them. These describe the way I was feeling or explain the reasoning behind an incident to help people better understand the lived experience behind the numbers. The motivation to design a data visualization had been to raise public awareness and understanding of OCD. The visualization, alongside an article about the potential for the medium of data visualization to make personal experiences of anxiety disorders more visible, was published in openDemocracy in May 2017. It was titled 'Visualising Mental Illness' (Simpson, 2017). 


\section{Data subjectivities}

By manually gathering, analysing, and visualizing my own data I was faced with some of the same conscious and unconscious decisions that data visualization designers encounter in their work (D'Ignazio \& Klein, 2016). The process was particularly revealing about the subjectivities that are at the heart of all datasets and their visual representation.

There is a labour involved in making data meaningful (Nafus, 2014). Yet, the people working behind the scenes to collect data, to store them, to analyse them, and to make them visible remain hidden to the audiences of visual representations of data (D'Ignazio \& Klein, 2016). Through the process of slow, manual, small-data gathering the labour of sense-making became visible to me. In collecting and analysing such personal data I connected with the emotive and embodied experience of conducting research (Law, 2017). At times I felt emotional while recording the data and, when reviewing them, I felt shocked at the number of incidences I had recorded. It seems to me that the emotive and embodied experiences of research undoubtedly shape data collection and the way they are analysed. Yet these experiences are almost always left out of data's visualization.

Kennedy \& Hill (2018) describe the importance of feelings and emotions when people who are not experts in data engage with and make sense of data visualizations. The process I undertook suggests that emotions can also play a significant role for the people who are working with data. I found collecting, analysing, and visualizing my data, so that they made sense to other people, required me to connect emotionally with the data and design process. Of course the extent to which design labour is emotional is dependent on the subject matter of the data and their relationship to it. Not only was the topic of my data visualization sensitive, it was extremely personal, and this does not reflect the majority of data visualization work.

There are, however, other examples of data visualizations which represent sensitive topics and whose design prompts emotional engagement. Digital interactive examples include Valentina D'Efilippo's (n.d.) Poppy Field (a beautiful and poignant representation of those who have lost their lives in wars during the nineteenth century) and Periscopic's (n.d.) hard-hitting visualization Gun Killings in the U.S. (a visualization of the lives lost and years stolen by gun violence). While the data illustration work of Mona Chalabi (n.d.) and Dear Data (n.d.) demonstrate how data can be represented with warmth and a sense of connection to the person who designed the visualization. These examples add weight to the argument that the embodied and emotional labour required to make sense of data should 
be considered as part of the lived experience of data visualization. Being open about these aspects of data visualization design makes it possible to appreciate the situated nature of data visualizations. In recognizing this, the idea that there are multiple alternative truths within data might become more apparent to those viewing a data visualization (D'Ignazio \& Klein, 2016).

In order for the visualization to tell a coherent story about my personal experience of OCD I was faced with choices. These involved what data to include, what data to leave out, which characteristics of the data to conceal, and which to make visible, so that the visualization makes sense to another person. Data visualizations are always partial, they can never represent all that a dataset might have to tell us (Boehnert, 2015). It is generally accepted that in order to present data graphically, it is necessary to discard much of what characterizes the data through processes of reduction (Manovich, 2011). Looking through my own data I made the decision to focus on one main theme in order to tell a coherent narrative; my fear of losing information. Every time I repeatedly checked the gas hob was turned off, for example, or the iron unplugged, was excluded from the visualization. In order to be transparent about the missing data I included a handwritten annotation acknowledging the missing data points. This kind of contextual detail helps to make visible both the situated nature of data visualization and the subjectivities of data visualization designers, by hinting at the ways in which their work might be influenced by their own decision-making (D'Ignazio \& Klein, 2016).

\section{The circulation of data visualization}

When 'Visualising Mental Illness' (Simpson, 2017) was first published it featured as the lead story on the home page of openDemocracy. The front page feature led with the title, a one-line summary and a large image of the visualization with a click-through link to the full article. The hand-drawn visualization stood out among the digital photographs which accompanied many of the other articles. As with all the stories they publish, openDemocracy tweeted about the article to their followers who number over 60,000 . My colleagues and friends retweeted openDemocracy's tweets, and composed their own to share the article, often embedding an image of the visualization into their tweets. It was a surreal experience watching this deeply personal visualization, designed in my flat in the North of England, spread across the world via social media. 
As I watched my data visualization circulate on Twitter I became very aware that I had no control over who shared the image or the message they attached to it. This made me feel uncomfortable; this was my data, my story, and yet it was being repurposed and circulated to support other people's agendas. John Berger (1972) argued that once an artwork has been reproduced it becomes a form of information. People can use a reproduced artwork to support their own arguments, which may differ from how the artist intended their work to be interpreted (Berger, 1972). Although Berger is talking about the art world, it is interesting to draw on him to think about how the meanings of data visualizations are also not fixed. Through the digital reproduction and circulation of this hand-drawn data visualization, it lost some of its original intended meaning and gained new meanings. This has implications when thinking about the politics of data visualizations as they circulate through social networks. Data and their visualization are always bound up in politics and power relations, which are played out in the kinds of data collected and what, from that data, is made visible (Boehnert, 2015; Kennedy \& Hill, 2017). The way in which people might repurpose the information to fit their own political agendas adds another layer of complexity in unpacking the ideological work that data visualizations do (Kennedy \& Hill, 2017, p. 773).

\section{The politics of hand drawing}

Thanks to its circulation on Twitter, the article was picked up by Scientific American in the US, which featured the data visualization on its visual blog. It also caught the attention of a national newspaper in Australia which featured the visualization in an online article on mental ill health. Significantly, the publication of the article in openDemocracy coincided with Mental Health Awareness Week in the UK and Mental Health Month in the US. Its timely publication may explain some of the interest in the visualization on social media and by the aforementioned publications. Nevertheless, in a field dominated by digital design it was surprising to see this static, hand-drawn, small-data visualization capture people's attention. This has led me to consider the significance of the medium of hand drawing in producing alternative visual representations of data.

Digital data visualizations are presented as complete and neutral reflections of data. The organizational conventions that data visualization designers draw on play an important role in making them appear objective (Kennedy, Hill, Aiello, \& Allen, 2016). This is in sharp contrast to the way 
in which hand-drawn images are more often presented as imperfect and incomplete representations of a concept (Dexter, 2005). Rather than being associated with technical neutrality, drawing appears subjective and linked to qualities like 'intimacy, informality, and authenticity' (p. 5). The ways in which drawing is perceived to be subjective hints at the work digitalization does in portraying data visualizations as objective, technical, and accurate. Therefore, at first, drawing may appear to be incompatible with data visualization. However, drawings are particularly good at expressing 'emotion, experience, and feeling', all important elements in developing a narrative (p. 8). Using affect in design is an important strategy in engaging people in an issue and one that is utilized by some data visualization designers (D'Ignazio \& Klein, 2016). Indeed, many designers recognize that good data visualization design provokes feeling in their audiences (Kennedy \& Hill, 2018).

In hand drawing my visualization, its imperfections were made visible. For example, the wobbly circles representing individual data points, or the rubbed out pencil lines still faintly visible in the background. Dexter (2005) argues that it is the visibility of such mistakes that gives drawing an air of authenticity and honesty. These imperfections, combined with the personal nature of the data and the handwritten annotations, made for a powerful and affective data visualization, which captured people's attention. Yet, the notion of authenticity troubles me. Although the subjectivities of my small-data visualization might appear to be more visible, it is not necessarily more honest than a digital graphical expression of data.

Using hand-drawn images to communicate serious topics is nothing new. In the field of comic journalism, graphic representations in a comic's style are used to depict 'hard news' (Weber \& Rall, 2017, p. 382). In their research, Weber and Rall (2017) identified several strategies that comic journalists use to give their work authenticity, including the use of 'visual stylistic devices' (p. 386). Citing the use of colour, mark making and handwritten text, they explain how visual styles help to 'remind the reader' that the comic is a subjective representation, made by the author (p. 386). Indeed, Weber and Rall (2017) say how comic journalism's obvious subjectivity makes it appear transparent and honest. They note how hand drawings produce a sense of honesty as they make readers aware that they represent the author's interpretation of an event.

In the hand-drawn visual style of my own data visualization I subconsciously adopted many of the authenticity strategies which Weber and Rall (2017) identified in their research. These are in contrast to the visual strategies and conventions used by digital data visualization designers, which 
often work to portray a sense of objectivity (Kennedy et al., 2016). However, I find it hard to argue that either a digital or hand-drawn approach is inherently more or less honest, even though honesty is a quality associated with the authenticity of the hand-drawn. Although one visual style might appear more authentic, does this mean it is more honest? This raises questions around claims to authenticity and honesty which are communicated through the visual style of data visualizations. These questions are significant in the context of the ideological power of data visualizations and the politics embedded within them (Boehnert, 2015; Kennedy \& Hill, 2017). As David Beer (2013) argues, 'There is something convincing about visuals, however it is that they have been created' (p. 118). Although the hand-drawn nature of the data visualization I designed suggests the subjectivities within the data collection and design process, the decisions I made which have shaped my representation of the data remain hidden. Perhaps then, we need to consider extending our conversation about the politics of data visualizations to explicitly include analogue, hand-drawn, designs.

\section{Conclusion}

The subjectivities of data and their visual representation tend to be obscured in digital data visualization design. Although research suggests that designers are aware that the design process involves decisions which will prioritize certain viewpoints of the data, the conventions they work within play a role in communicating an 'aura of objectivity' (Kennedy et al., 2016, p. 723). Making the subjectivities of visual representations visible to audiences through the medium of hand drawing can work to imply authenticity (Weber \& Rall, 2017). However, just because data subjectivities appear more visible it should not be assumed that the visual representation is any more honest. The situated nature of data and their visualization always shapes their design in ways that are invisible to the audience. The design process I embarked upon required methods of selection and reduction which produced a very particular view of the data. This complemented the story I wanted to tell, while alternative possible narratives within the data remained hidden. Developing ways of making these design decisions more visible to audiences might work to unpack some of the ideological power data visualizations possess (Kennedy \& Hill, 2017), by introducing the idea that multiple alternative truths might exist within the data (D'Ignazio \& Klein, 2016).

By borrowing from elements of personal criticism and critical making, this chapter has brought theoretical and conceptual ideas into a personal 
context. In doing so it has revealed the different ways in which data can be embodied, emotive, and felt (Kennedy \& Hill, 2018). This supports Kennedy and Hill's (2018) argument that we must look beyond technological structures, to consider data visualization as experienced as part of everyday life. Critically engaging with my own experience of visualizing mental illness has demonstrated how existing conceptual ideas can be interrogated, and new ones emerge, when data visualizations are explored as a lived experience.

\section{References}

BBC (2017, February 26). Disability benefits: PIPs should be for 'really disabled'. $B B C$. Retrieved from https://www.bbc.co.uk/news/uk-39097019

Beer, D. (2013). Popular culture and new media: The politics of circulation. Basingstoke: Palgrave Macmillan.

Berger, J. (1972). Ways of seeing. London: Penguin Books Ltd.

Boehnert, J. (2015). Viewpoint: The politics of data visualization. Retrieved August 3 , 2016 from http://discoversociety.org/2015/08/03/viewpoint-the-politics-of-datavisualization/

boyd, d., Crawford, K. (2012). Critical questions for big data: Provocations for a cultural, technological and scholarly phenomenon. Information, Communication and Society, 15(5), 662-679.

Chalabi, M. (n.d.) Mona Chalabi. Retrieved October 30, 2018 from https://monachalabi.com/illustrations/

Crawford, K. (2013). The Hidden Biases in Big Data. Retrieved June 9, 2016 from https://hbr.org/2013/04/the-hidden-biases-in-big-data

Dear Data (n.d.). Dear data. Retrieved September 4, 2016 from http://www.dear-data. com/theproject

D'Efilippo, V. (n.d.) Poppy field. Retrieved October 30, 2018 from http://www. valentinadefilippo.co.uk/projects/poppy-field/

Dexter, E. (2005). Vitamin D: New perspectives in drawing. London: Phaidon Press Limited.

D'Ignazio, C., \& Klein, L. (2016, October). Feminist Data Visualization. Paper presented at and published in the workshop proceedings from the Workshop on Visualization for the Digital Humanities at IEEE VIS Conference, Baltimore, Maryland.

Kennedy, H., Hill, R. L., Aiello, G., \& Allen, D. (2016). The work that visualisation conventions do. Information, Communication and Society, 19(6), 715-735. https:// doi.org/10.1080/1369118X.2016.1153126

Kennedy, H., Hill, R. L. (2017). The pleasure and pain of visualising data in times of data power. Television and New Media, 18(8), 769-782. 
Kennedy, H., Hill, R. L. (2018). The feelings of numbers: Emotions in everyday engagements with data and their visualisation. Sociology, 52(4), 830-848. https:// doi.org/10.1177/0038038516674675

Kitchin, R. (2014). The data revolution: Big data, open data, data infrastructures and their consequences. London: Sage Publications Ltd.

Law, J. (2016). Modes of knowing: Resources from the Baroque. In: J. Law \& E. Ruppert (Eds.), Modes of knowing: Resources from the Baroque. (pp. 17-56). Manchester: Mattering Press.

Manovich, L. (2011). What is visualization? Visual Studies, 26(1), 36-49.

Miller, N. K. (1991). Getting personal: Feminist occasions and other autobiographical acts. London: Routledge.

Nafus, D. (2014). Stuck data, dead data, and disloyal data: The stops and starts in making numbers into social practices. Distinktion:Journal of Social Theory, 15(02), 208-222.

Periscopic (n.d.) U.S. gun deaths in 2013. Retrieved October 30, 2018 from https:// guns.periscopic.com/?year=2013

Ratto, M. (2011). Critical making: Conceptual and material studies in technology and social life. The Information Society, 27(4), 252-26o.

Ratto, M., Wylie, S. A., Jalbert, K. (2014). Introduction to the special forum on critical making as research program. The Information Society, 3o(2), 85-95.

Simpson, J. (2017, May 10). Visualising mental illness. openDemocracy. Retrieved from https:/www.opendemocracy.net/transformation/jill-simpson/ visualising-mental-illness

Stanley, L. (1992). The auto/biographical I. Manchester: Manchester University Press.

Stanley, L., Wise, S. (1983). Breaking out: Feminist consciousness and feministresearch. London: Routledge \& Kegan Paul plc.

Ursyn, A. (2008). Digital drawing, graphic storytelling and visual journalism. In: S. Garner (Ed.), Writing on drawing: Essays on drawing practice and research. (pp. 169-177). Bristol: Intellect Books.

Weber, W., Rall, H.-M. (2017). Authenticity in comics journalism: Visual strategies for reporting facts. Journal of Graphic Novels and Comics, 8(4), 376-397.

\section{About the author}

Jill Simpson is a doctoral researcher in the Sociology Department at the University of York. Her research explores the meaning making processes at work in the design and interpretation of data visualizations. More broadly Jill's research interests combine critical data studies, interdisciplinary social research, and public engagement through creative practice. 